UCRL-ID-118940

\title{
Descriptions of Positron Defect Analysis Capabilities
}

Richard H. Howell

Lawrence Livermore National Laboratory

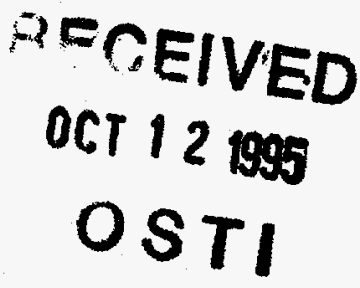

Livermore, California 94551

October 1994

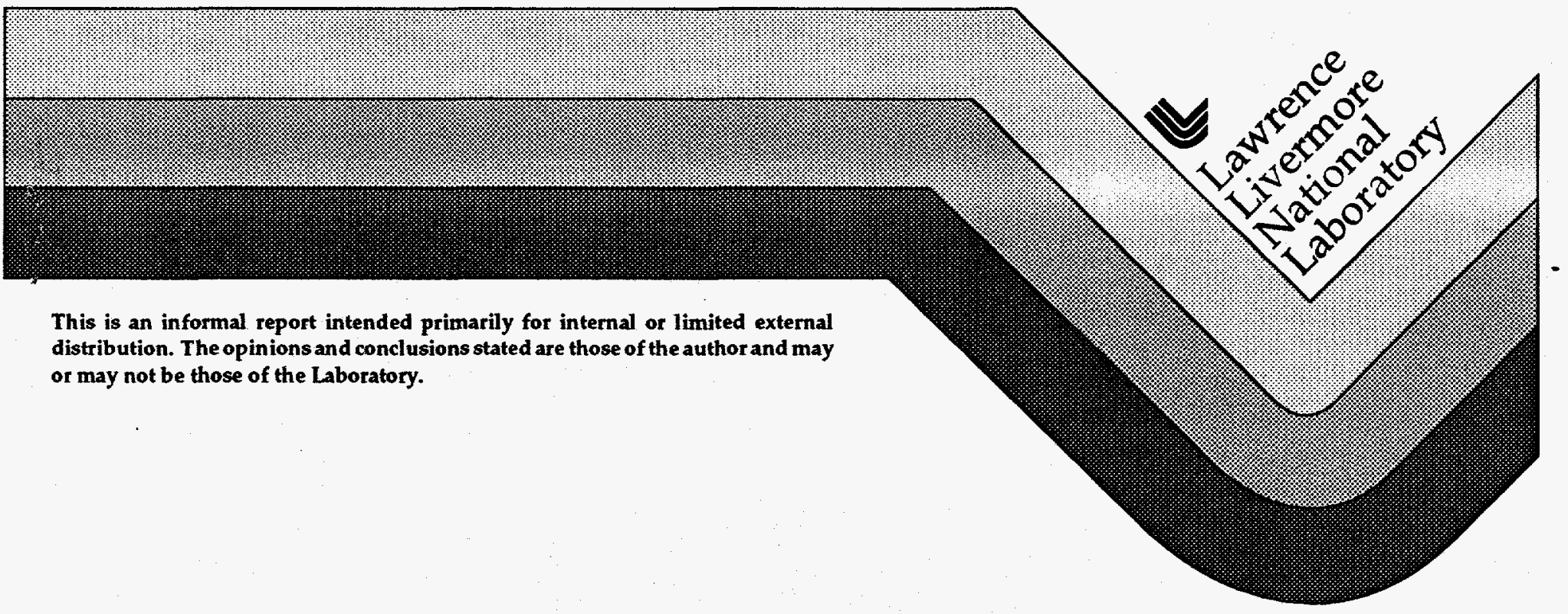




\section{DISCLAMER}

This document was prepared as an account of work sponsored by an agency of the United States Covernment. Neither the United States Covernment nor the University of California nor any of their employees, makes any warranty, express or implied, or assumes any legal liability or responsibility for the accuracy, completeness, or usefulness of any information, apparatus, product, or process disclosed or represents that its use would not infringe privately owred rights. Reference herein to any specific commercial product, process, or service by trade name, trademark, manufacturer, or otherwise, does not necessarily constitute or imply its endorsement, recommendation, or favoring by the United States Covernment or the University of California. The views and opinions of authors expressed herein do not necessarily state or reflect those of the United States Covernment or the University of California, and stall not be used for advertising or product endorsement purposes.

This report has been reproduced directly from the best available copy.

Available to DOE and DOE contractors from the Office of Scientific and Technical Information

P.O. Box 62, Oak Ridge, TN 37831

Prices available from (615) 576-8401, FTS 626-8401

$$
\begin{aligned}
& \text { ! }
\end{aligned}
$$

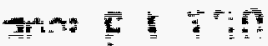

$$
\begin{aligned}
& \div \div
\end{aligned}
$$$$
\text { Available to the public from the }
$$$$
\text { National Technical Information Service }
$$$$
\text { U.S. Department of Commerce }
$$$$
5285 \text { Port Royal Rd. }
$$$$
\text { Springfield, VA } 22161
$$ 


\section{DISCLAIMER}

\section{Portions of this document may be illegible in electronic image products. Images are produced from the best available original document.}


Descriptions of positron defect analysis capabilities

\author{
Richard H. Howell
}

A series of descriptive papers and graphics appropriate for distribution to potential collaborators has been assembled. These describe the capabilities for defect analysis using positron annihilation spectroscopy. The application of positrons to problems in the polymer and semiconductor industries is addressed.

This work was performed under the auspices of the U.S. Department of Energy by the Lawrence Livermore National Laboratory under Contract No. W7405-ENG-48 


\section{An integrated analytical capability for open volume defects}

\section{Summary}

WNL is developing a facility that would both satisfy immediate analysis needs for defect analysis at shallow depths for films and interfaces and deep within thick samples. This facility would provide growing capabilities for a general user community in the coming decade. It contains three devices based on upgrades and extensions of existing capabilities using respectively a3MV electron Van de Graff, the intense positron beam at the electron linac and laboratory detector systems. Each of these devices meets or advances the state of the art in defect analysis and together they form an unrivaled defect analysis capability including rapid analysis of deeply distributed defects in engineering samples encased or covered by overlayers, microprobe analysis of shallow defects in thin films and at interfaces and the special identification of defects in bulk materials.

\section{Applications}

The role of defects in thin films and at interfaces is central to understanding the properties of many advanced materials of programmatic and commercial interest. For example the role of defects in the nucleation of voids in electromigration is pivotal in the development of better electronics, the molecular free volume elements in polymeric coatings is a determining factor in their mechanical and aging properties and the defect characteristics of metals and alloys determines their strength and corrosion resistance.

Defects are a generic problem that affect much of materials behavior. The evolution of defects during conditions of unusual temperature and pressure or over long periods of time determines the ultimate strength and corrosion of many systems. Consequently a broad range of problem ranging from identifying the defect species in high temperature superconductors or the defect active in the oxygen transport in uranium corrosion to the validation of bonds between metals require defect identification and quantification. Many of these problems are well suited to positron lifetime analysis which is a non-destructive technique with specific defect response. Two special problems of current national interest are described below.

\section{Semiconductor Materials Analysis}

Electrical properties of semiconducting materials and devices are determined by defects either purposefully engineered or other wise occurring. There is an ongoing need for non destructive analysis of defects for both research and production line quality assurance in the bulk of the material and particularly at interfaces in layers deposited for device fabrication. The utility of positron beam analysis for solving problems of interest to the semiconducting industry is recognized world wide with active groups in the US, Japan, Germany, Canada, and Finland. Positron annihilation analysis with a beam is a direct method, capable of measuring the depth distribution and microscopic characteristics of defects in semiconducting materials and structures. Measurement of the annihilation characteristics of mono-energetic positrons implanted at known depths can be related to the charge carrier diffusion, the interfacial defect concentrations and the defect population of thin layered materials. These measurements can provide unique data on systems such as ion implanted silicon, silicon epitaxially grown on silicon, silicon implanted by oxygen and stress voiding and electromigration. Positron annihilation lifetime measurements have been used to identify defect types and charge states in 
bulk semiconductor samples. These measurements can be extended to thin samples using a pulsed positron microbeam under design at LLNL.

\section{Polymer Free Volume Analysis}

Macroscopic and electrical properties of polymers can be understood using theories based on the free volume of the material. Application of the theory, which unifies the discussion of impact strength, elastic properties some electrical properties and aging has been limited by the lack of definitive measurements of the free volume characteristics in real materials. Positron annihilation analysis is capable of measuring the free volume fraction and microscopic size distribution in a non-destructive setting. By measuring either the lifetime of the positron in the material or the angular correlation of the annihilation gamma rays, positron annihilation analysis can determine the size and number of the free volume elements from sizes of 1 to over $1000 A^{3}$. The positron probe does not damage the sample or produce a major distortion of the free volume elements during measurement.

\section{Current Capabilities}

LLNL has a long and productive history in the innovative development and application of all major positron analysis spectroscopic techniques to problems in materials science and general physics. We are currently running state of the art equipment performing all major experimental techniques and have pioneered both intense positron beams and angular correlation spectrometers which are now copied world-wide. These have produced valuable data on the behavior of materials systems including: defect determinations in a variety of systems, unique demonstrations of the Fermi surface in several high temperature superconductors, and original studies on the utility of positron spectroscopy as a surface electronic structure probe. With these capabilities we can determine the free volume in thick samples of polymeric materials, in situ, under set conditions of temperature, pressure and mechanical stress. We can also determine the defect characteristics of semiconductor systems in a variety of environments.

In the laboratory positron lifetime spectroscopy is a proven analytical tool for the nondestructive analysis of defects and open volume elements in metals, semiconductors and polymeric materials. With positron lifetime spectroscopy we can detect and identify any defects containing an open space in which positrons can trap. In an extension of these techniques experimental analysis can be done at shallow depths with microprobe spatial resolution or at deep penetration for bulk analysis. In all cases the results can be understood by comparison to values obtained from first principles calculations. They have been recently used at LLNL to determine the defects in high temperature superconductors and are presently part of the LLNL-Boeing CRADA on accelerated aging in composite materials.

\section{Extensions of capabilities}

There is a national need, identified by the semiconductor and polymer industries and the positron research community, to perform all kinds of positron defect analysis in thin films, at buried interfaces and in the near surface regions of deposited materials. Measurement of the free volume in free standing thin films and coatings or the specific identification of semiconductor defects in deposited layers and at interfaces requires a pulsed, monoenergetic small diameter beam of positrons for implantation in the film material. At the DOE workshop held at Rancho Mirage in 1992, LLNL was identified as an institution of choice for early development of an apparatus available at the national level for performing defect specific, positron lifetime analysis. 
UNL proposes to fill the need for a US capability by developing a positron pulsed bearnmicroprobe for qualifying technologically important materials by measuring the depth dependence, spatial distribution and specific identity of free volume and defect populations. We propose such an apparatus to solve problems in polymeric coatings, semiconductor interconnects, defect distributions in deposited films and small size samples of energetic materials. The UNL facility will complement the activities of the National Positron Facility which will be included as part of the activities fo the Advanced Neutron Source and provide a unique national resource that will be technically superior to those found in Germany and Japan. In its final form a sub-micron positron beam capable of depth dependent defect analysis in the first few microns of a sample would be obtained. Development of the apparatus would be done in stages that will allow defects analysis at each level of capability.

Conventional laboratory analysis of deeply occurring defects in bulk samples is unable to fulfill current LLNL needs. Due to essential physical limitations only low throughput with significant sample geometry restrictions can be obtained in conventional laboratory experiments. We will obtain higher throughput and a significant easing of sample geometry restrictions by performing these analyses with a positron beam accelerated in our 3MV Van de Graff in using a system modeled on that in use in Germany. In this apparatus, defect analysis in engineering samples of encased materials can be performed on up to 100 samples a day.

Confirmation of positron lifetime defect identification may be necessary by other techniques. Positron momentum measurements measured by angular correlation techniques can be performed in a state of the art spectrometer. At LLNL diffuse $x$-ray scattering and other defect sensitive techniques, is currently available.

\section{LLNL Experience}

The accelerator based positron beam-lifetime analysis enables us to fill an immediate need in broad areas of interest to DOE and US industry. LLNL is the ideal site for a US experimental program because of its technical capabilities in positron analysis, its the strong materials science base and associated laboratory experiments. Present levels of linac positron beam intensity are sufficient for bunching and multiple stages of beam focusing so that customized experiments can be performed and all the facilities are easily accessible to researchers. Accelerator based positron beams, such as those at LLNL, are inherently more reliable than intense beams dependent on radioactive sources and the use of a timed positron beam for lifetime measurements is critical. The combination at a single site of the shallow and deep defect analysis experimental capabilities, supported by first principle calculations, is unique and puts LLNL at the forefront of defect related materials reliability research world wide. The LLNL positron and linac programs are experienced in working with diverse industrial clients in a Cooperative Research and Development Agreement format and CRADA related activities are a substantial fraction of the present accelerator and positron measurement activities.

For more information please contact, Richard H Howell, L 280, Lawrence Livermore National Laboratory, Livermore Ca 94550, 510-422-1977, FAX 510-422-7300. 


\section{FACT SHEET}

Positron lifetime microprobe.

Major technical contributor:

Lawrence Livermore National Laboratory

Initial development period :

Two years from beginning of design to first positron microbeam-lifetime operation

Cost:

1.5M\$ over two years for equipment, personnel and linac operations expenses during development. $300 \mathrm{~K} \$ / \mathrm{yr}$. operations after that for experiments.

Major equipment:

LLNL electron linac, Intense positron beam, apparatus and electronics.

Milestones:

Construct a pulsed positron beam with pulses less than 100 Ps duration and associated detection system.

Construct a pulsed beam with micron scale spot size and variable energy from $100 \mathrm{eV}$ to $25 \mathrm{keV}$ and associated detection and alignment system.

Obtain data on model systems to demonstrate correlation of specific defects with critical materials properties.

Obtain data to discriminate among competing theoretical defect based explanations of critical phenomena.

Potential uses:

Defects are a controlling factor in much of the electrical and mechanical response of materials in this scale range. Often the specific role of defects is only suggested since direct observation is difficult with existing techniques. With data from a positron beam lifetime microprobe, defect based models of materials behavior can be verified or negated. The instrument will allow depth profiling of defects in thin over layers and at interfaces with defect specific analysis. Using this tool we can determine the concentration and identity of defects controlling materials properties and transport in model systems. Changes in the defect population can be followed in detail and both changes in type and concentration of the defects can be simultaneously determined.

Major competitors:

US. none

World wide, ETL, Tsukuba Japan, Hochschule der Bundeswehr, Neubiberg, Germany 


\section{FACT SHEET}

Positron lifetime deep defect probe.

Major technical contributor:

Initial development period :

Cost:

Major equipment:

Milestones:

Potential uses:

Major competitors:
Lawrence Livermore National Laboratory

Six months from beginning of design to first positron beam-lifetime operation

$100 \mathrm{~K} \$$ for development, equipment, personnel and Van de Graff operating expenses. $100 \mathrm{~K} \$$ operations after that for experiments.

3 MV electron Van de Graff, Lifetime apparatus and electronics.

Construct a positron beam lifetime spectrometer at 200 Ps resolution for use with $3 \mathrm{MeV}$ positrons.

Construct a $3 \mathrm{MeV}$ positron beam by inserting a thermalized positron source in the terminal of the Van de Graff.

Obtain data on model systems to demonstrate correlation of specific defects with critical materials properties.

Obtain data to discriminate among competing theoretical defect based explanations of critical phenomena.

Due to the high sample throughput and minimal restrictions on sample geometry this apparatus will be the preferred defect and free volume analysis instrument. The instrument will allow analysis of defects in engineering samples and encased samples with defect specific analysis. Using this tool we can determine the concentration and identity of defects controlling materials properties and transport in model systems and formed parts. Changes in the defect population can be followed in detail and both changes in type and concentration of the defects can be simultaneously determined.

US. none

World wide, Max Plank Institute, Stuttgart, Germany 


\section{Polymer Free Volume Analysis}

Need for Polymer Free Volume Analysis Macroscopic and electrical properties of polymers can be understood using a theory based on the free volume of the material. Application of this theory, which unifies the discussion of impact strength, elastic properties some electrical properties and aging has been limited by the lack of definitive measurements of the free volume characteristics in real materials. There is now a new direct method, positron annihilation analysis, capable of measuring the free volume fraction and microscopic size distribution in a non-destructive setting. By measuring either the lifetime of the positron in the material or the angular correlation of the annihilation gamma rays, positron annihilation analysis can determine the size and number of the free volume elements from sizes of 1 to over $1000 \AA^{3}$. The positron probe does not produce a major distortion of the free volume elements during measurement.

Present capabilities We at LLNL have a long and productive history in the innovative development and application of positron techniques to problems in materials science and general physics. We are currently running state of the art equipment performing all major positron analysis experiments including positron annihilation lifetime, angular correlation and beam profiling measurements. This capability is based on the pioneering development of both intense positron beams and angular correlation spectrometers which are now widely copied at other institutions world-wide. There are two new accelerator based positron capabilites at LLNL. One for deep probing of thick samples is just completed and one for near surface measurements is under development. With the developed capabilities we can determine the free volume in thick engineering samples of polymeric materials in situ under set conditions of temperature, pressure and mechanical stress or changes in the free volume as a result of sample modification due to exposure to light or aging under in electric fields

Thin film measurements Measurement of the free volume in free standing thin films and coatings requires a monoenergetic beam of positrons that are implanted in the film material. We are now developing a positron lifetime beam-microprobe for materials analysis by coupling three major successes of positron materials analysis: positron lifetime determination of defect properties, defect depth profiling by variable energy positron beams, and microscopic beam dimensions by remoderation. With this apparatus free volume and defect populations would be measured on small sample area as a function of depth from the surface. Presently, positron lifetime measurements from a beam are available only for large size beams, $-1 \mathrm{~cm}$, and only in Germany and Japan. US researchers and industrial labs needing positron beam-lifetime analysis are required to analyze only large sample features and to send their samples to a foreign laboratory.

LLNL Facilities and Experience Accelerator based positron beam-lifetime are enabling and fill an immediate need in broad areas of interest to DOE and US industry. The LLNL positron beam is the ideal site for a US experimental program because of its the strong materials science base and associated laboratory experiments. Present levels of linac positron beam intensity are sufficient for bunching and multiple stages of beam focusing so that customized experiments can be performed and the facilities are easily accessible to researchers without undue security restrictions. Accelerator based positron beams, such as those at LLNL, are inherently more reliable than intense beams dependent on radioactive sources and the use of a timed beam for lifetime measurements is critical.

For more information please contact, Richard H Howell, L 280, Lawrence Livermore National Laboratory, Livermore Ca 94550, 510-422-1977, FAX 510-422-7300. 
LLNL provides an integrated capability for defect analysis analysis and materials modification

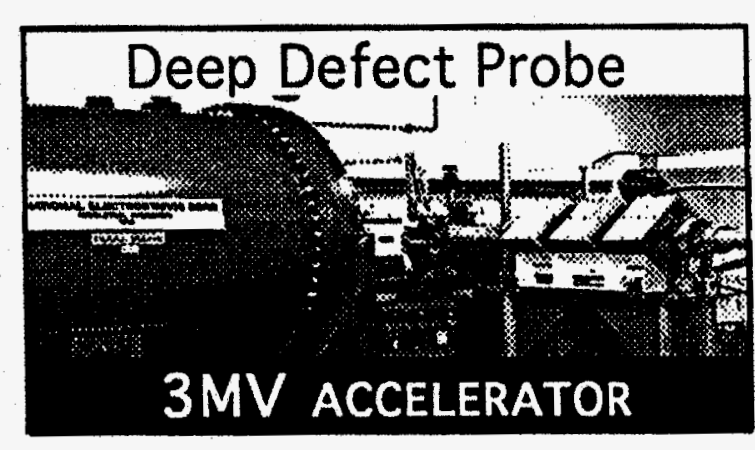

Low E, Pulsed,

Shallow Defect Microprobe

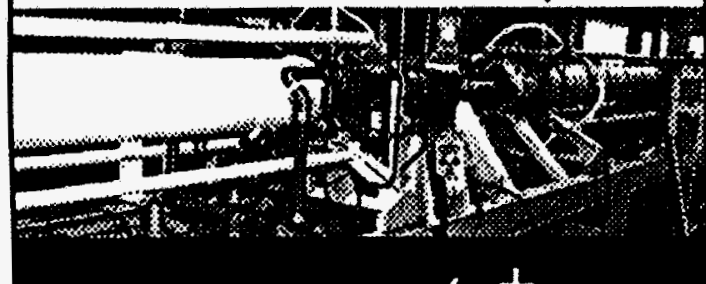

ELECTRON LINAC/ $\mathrm{e}^{+}$BEAM

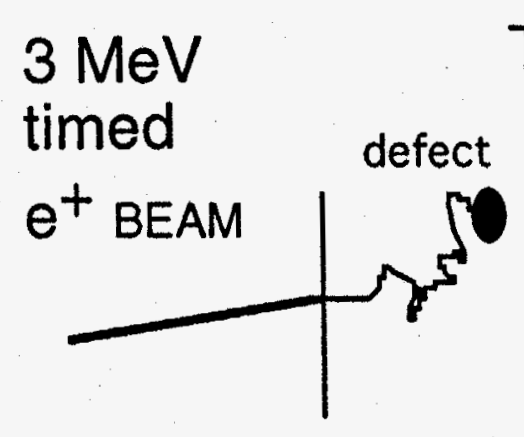

Trapped e+ has unique lifetime

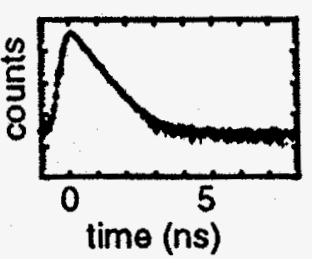

Vacancies

Voids

Dislocations

Charge state

Defect specific analysis $\mathrm{mm}$ depth sampling

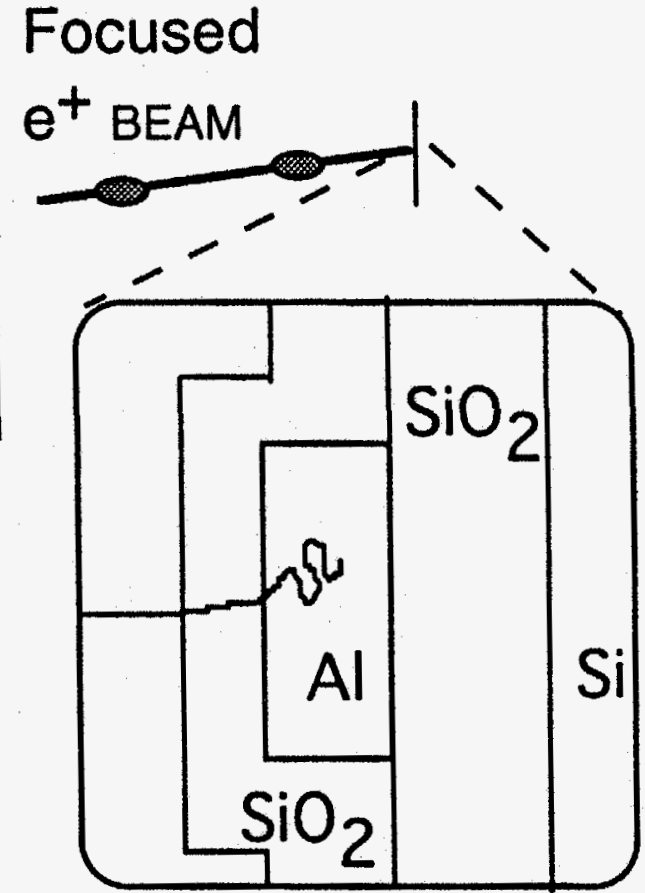

Surface to micron depth scanning

Analysis of interconnects at device level organization

Sub micron lateral and depth resolution 
New measurements are enabled by the development of a new lifetime spectrometer in B194 for composite materials

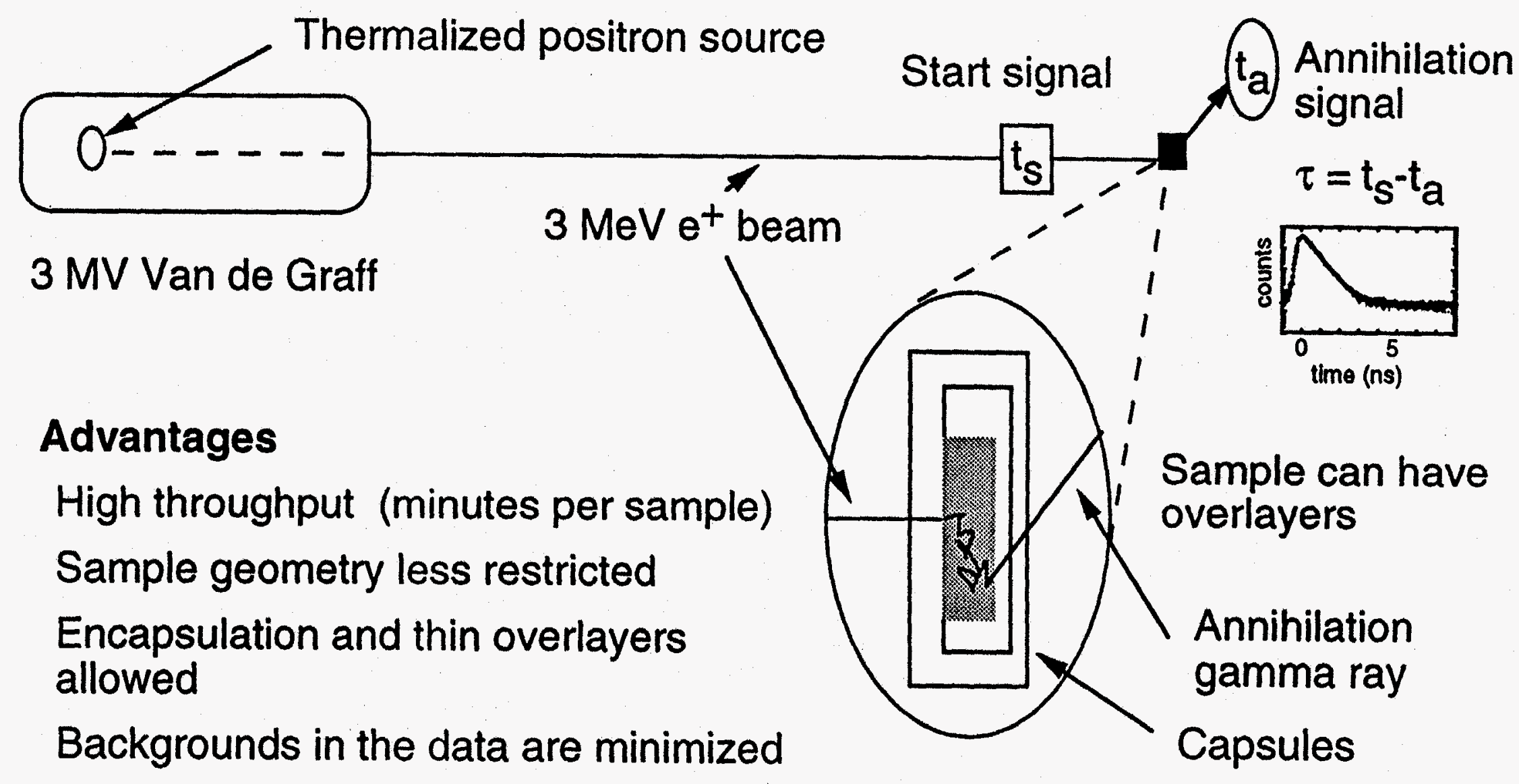

Extreme in situ conditions available, $\sim 0 \mathrm{~K}$ to melting 
The Linac-Positron Facility Provides An Integrated Capability In Plasma Physics, Fundamental Physics, Applied Physics, Materials Analysis And Materials Modification

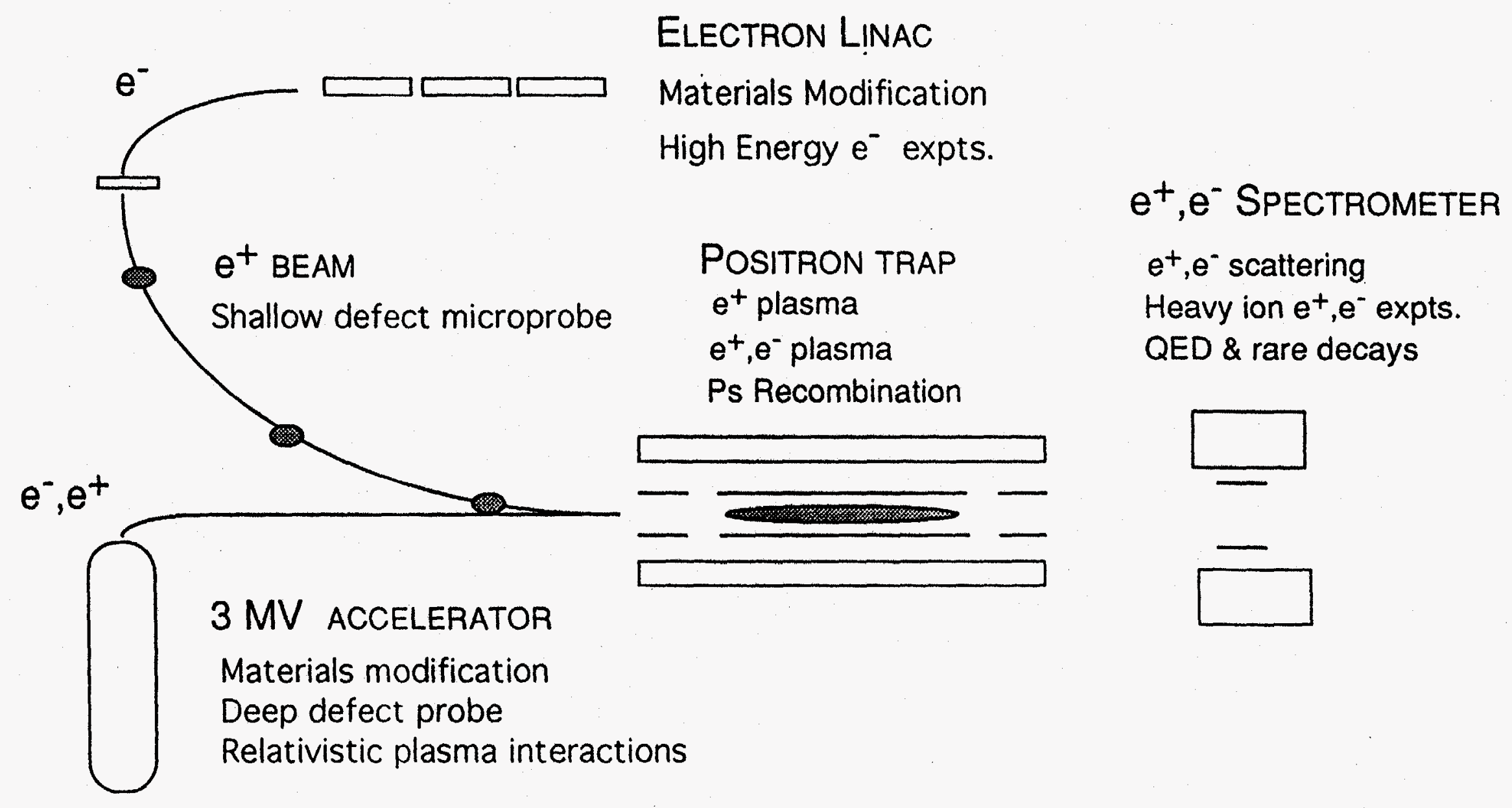

